\title{
On Cohomology Theories of Infinite CW-complexes, II
}

By

\author{
Zen-ichi Yosimura
}

In the first paper under the same title ( $[\mathrm{I}]$ in the references at the end of the paper) we discussed main strong convergence conditions of spectral sequences associated with an additive cohomology theory $h$. In the present paper we discuss mainly conditions when $h(X)$ is Hausdorff.

We understand by a cohomology theory $h$ a general reduced cohomology theory defined on the category of based $C W$-complexes. Let $X$ be a based $C W$-complex and $\mathfrak{u}_{X}=\left\{X^{\lambda}\right\}$ the set of all finite subcomplexes of $X$. The subgroups $F^{\lambda} h^{n}(X)=\operatorname{Ker}\left\{h^{n}(X) \rightarrow h^{n}\left(X^{\lambda}\right)\right\}$ of $h^{n}(X)$ give $h^{n}(X)$ the structure of a topological abelian group. In general $h^{n}(X)$ is not Hausdorff.

Under the assumption that each skeleton $X^{p}$ of $X$ is a finite $C W$. complex, Buhštaber and Miščenko [3] and Landweber [13] investigated conditions that $h(X)$ is Hausdorff in case $h=K$, complex $K$-theory, and $h=M U$, complex cobordism, respectively. In the present work we try to drop the above finiteness assumption on $X$ and investigate the conditions that $h(X)$ is Hausdorff for a general $h$. However, in the present discussions we must restrict our interest to the case that $h$ is additive and of finite type.

First we give a characterization of the closure of zero in $h^{n}(X)$ denoted by $S^{n}(X)$ (Theorem 2). In [3] the same characterization was given in case $h=K$, complex $K$-theory, by a different method.

In [2] we introduced classifying spaces of direct systems of based

Received July 6, 1972.

Communicated by N. Shimada.

* Department of Mathematics, Osaka City University, Osaka, Japan. 
$C W$-complexes and cellular maps which behave as a generalization of telescope constructions. By using the classifying spaces we construct co-Moore spaces $\check{M}$ and $S_{l}$ of type $(\hat{Z}, 2)$ and $(\hat{Z} / Z, 2)$ respectively, and define cohomology theories $h(; \hat{Z})$ and $h(; \hat{Z} / Z)$ following Dold [11]. Then the long exact sequence

$$
\rightarrow h^{n}(\quad) \rightarrow h^{n}(\quad ; \hat{Z}) \rightarrow h^{n}(\quad ; \hat{Z} / Z) \rightarrow h^{n+1}(\quad) \rightarrow
$$

associated with the coefficient sequence $0 \rightarrow Z \rightarrow \hat{Z} \rightarrow \hat{Z} / Z \rightarrow 0$ is obtained. This exact sequence is closely related to Hausdorff-ness of $h(X)$ (Theorem 3 ). Consequently we get necessary and sufficient conditions that $h(X)$ is Hausdorff (Theorems 4 and 5), cf., [3] and [13].

Finally we generalize Dold's Theorem [11] (Theorem 6). This theorem applied to $h(; \hat{Z} / Z)$ gives a sufficient condition that $h(X)$ is Hausdorff (Theorem 7).

We quote the theorem of $[\mathrm{I}]$ in such a form as "Theorem I.3" without any more mention.

\section{Inverse Limit Functor}

1.1. Inverse limit functor may not commute with the functors Ext and Tor in general. In order to supply this deficiency we need the following results (1.1), (1.2) and (1.3) due to Roos $[8,14]$.

Fix a (commutative) ring $\Lambda$ and a partially ordered set $I$. Let $M$ be a $A$-module and $\mathscr{A}=\left\{A_{\alpha}\right\}$ and $\mathscr{B}=\left\{B_{\alpha}\right\}$ be inverse and direct systems of $\Lambda$-modules over $I$ respectively.

(1.1) Assume that $I$ is directed. There is a strongly convergent spectral sequence $\left\{E_{r}\right\}$ associated with $\mathrm{Ext}_{\Lambda}^{*}\left(\varliminf_{\alpha}, M\right)$ such that

$$
E_{2}^{p, q}=\varliminf^{p} \operatorname{Ext}_{\Lambda}^{q}\left(B_{\alpha}, M\right)
$$

(1.2) There are two strongly convergent spectral sequences $\left\{E_{r}\right\}$ and $\left\{\bar{E}_{r}\right\}$ associated with the same graded $\Lambda$-module such that

$$
E_{2}^{p, q}=\varliminf^{p} \operatorname{Ext}_{A}^{q}\left(M, A_{\alpha}\right) \text { and } \bar{E}_{2}^{p, q}=\operatorname{Ext}_{A}^{p}\left(M, \varliminf^{q} A_{\alpha}\right)
$$


(1.3) Assume that $\Lambda$ is Noetherian and $M$ is finitely generated. There are two spectral sequences $\left\{E_{r}\right\}$ and $\left\{\bar{E}_{r}\right\}$ associated with the same graded 1-module such that

$$
E_{2}^{p, q}=\varliminf^{p} \operatorname{Tor}_{-q}^{\Lambda}\left(M, A_{\alpha}\right) \text { and } \bar{E}_{2}^{p, q}=\operatorname{Tor}_{-p}^{\Lambda}\left(M, \varliminf^{q} A_{\alpha}\right) \text {. }
$$

Remark. i) Instead of assuming that $\Lambda$ is Noetherian and $M$ is finitely generated we may assume that $M$ has a projective resolution by finitely generated $\Lambda$-modules.

ii) When the global dimension of $\Lambda$ is finite, the spectral sequences of (1.3) are strongly convergent.

(1.1)-(1.3) are easily proved by standard arguments about the spectral sequences associated with double complexes.

Here we restrict to the case $\Lambda=Z$ (the ring of integers). As corollaries of (1.1)-(1.3) we have the following (1.4)-(1.6).

Let $M$ be an abelian group and $\mathscr{A}=\left\{A_{\alpha}\right\}$ and $\mathscr{B}=\left\{B_{\alpha}\right\}$ be inverse and direct systems of abelian groups over $I$.

(1.4) Assume that I is directed. There are isomorphisms

$$
\varliminf \operatorname{Hom}\left(B_{\alpha}, M\right) \cong \operatorname{Hom}\left(\varliminf_{\alpha} B_{\alpha}, M\right)
$$

and

$$
\varliminf^{n} \operatorname{Ext}\left(B_{\alpha}, M\right) \cong \varliminf^{n+2} \operatorname{Hom}\left(B_{\alpha}, M\right) \quad \text { for } n \geqq 1
$$

and an exact sequence

$$
\begin{aligned}
0 \rightarrow \varliminf^{1} \operatorname{Hom}\left(B_{\alpha}, M\right) \rightarrow \operatorname{Ext}\left(\varliminf_{\alpha} B_{\alpha}, M\right) \\
\quad \rightarrow \varliminf \operatorname{Ext}\left(B_{\alpha}, M\right) \rightarrow \varliminf^{2} \operatorname{Hom}\left(B_{\alpha}, M\right) \rightarrow 0 .
\end{aligned}
$$

(1.5) There are abelian groups $\left\{H^{n}\right\}_{n \geqq 0}$ such that

$$
0 \rightarrow \operatorname{Ext}\left(M, \varliminf^{n-1} A_{\alpha}\right) \rightarrow H^{n} \rightarrow \operatorname{Hom}\left(M, \varliminf^{n} A_{\alpha}\right) \rightarrow 0
$$

and

$$
\rightarrow H^{n+1} \rightarrow \varliminf^{n} \operatorname{Ext}\left(M, A_{\alpha}\right) \rightarrow \varliminf^{n+2} \operatorname{Hom}\left(M, A_{\alpha}\right) \rightarrow H^{n+2} \rightarrow
$$


are exact, and in particular

$$
H^{0} \cong \varliminf \operatorname{Hom}\left(M, A_{\alpha}\right) \cong \operatorname{Hom}\left(M, \varliminf A_{\alpha}\right) .
$$

(1.6) Assume that $M$ is finitely generated. There are abelian groups $\left\{H^{n}\right\}_{n \geqq-1}$ such that

$$
0 \rightarrow M \otimes \varliminf^{n} A_{\alpha} \rightarrow H^{n} \rightarrow \operatorname{Tor}\left(M, \varliminf^{n+1} A_{\alpha}\right) \rightarrow 0
$$

and

$$
\rightarrow H^{n} \rightarrow \varliminf^{n}\left(M \otimes A_{\alpha}\right) \rightarrow \varliminf^{n+2} \operatorname{Tor}\left(M, A_{\alpha}\right) \rightarrow H^{n+1} \rightarrow
$$

are exact, and in particular

$$
H^{-1} \cong \varliminf \operatorname{Tor}\left(M, A_{\alpha}\right) \cong \operatorname{Tor}\left(M, \varliminf A_{\alpha}\right) .
$$

Using the functors Tor and Ext various functors of three variable are obtained by composition. We recall relations held between them.

Let $\Lambda$ be a ring and $L, M$ and $N$ be $\Lambda$-modules.

(1.7) There are two strongly convergent spectral sequences $\left\{E_{r}\right\}$ and $\left\{\bar{E}_{r}\right\}$ associated with the same graded A-module such that

$$
E_{2}^{p, q}=\operatorname{Ext}_{\Lambda}^{p}\left(M, \operatorname{Ext}_{A}^{q}(N, L)\right) \text { and } \bar{E}_{2}^{p, q}=\operatorname{Ext}_{\Lambda}^{p}\left(\operatorname{Tor}_{q}^{A}(M, N), L\right)
$$

(1.8) Assume that $\Lambda$ is Noetherian and $M$ is finitely generated. There are two spectral sequences $\left\{E_{r}\right\}$ and $\left\{\bar{E}_{r}\right\}$ associated with the same graded 1 module such that

$$
E_{2}^{p, q}=\operatorname{Ext}_{\Lambda}^{p}\left(\operatorname{Ext}_{\Lambda}^{-q}(M, N), L\right) \text { and } \bar{E}_{2}^{p, q}=\operatorname{Tor}_{-p}^{A}\left(M, \operatorname{Ext}_{\Lambda}^{q}(N, L)\right) .
$$

We remark that the spectral sequences of (1.8) are strongly convergent when the global dimension of $\Lambda$ is finite.

When we restrict to the case $\Lambda=Z$, the following results are immediately obtained.

Let $L, M$ and $N$ be abelian groups.

(1.9) There are isomorphisms 


$$
\operatorname{Hom}(M, \operatorname{Hom}(N, L)) \cong \operatorname{Hom}(M \otimes N, L)
$$

and

$$
\operatorname{Ext}(M, \operatorname{Ext}(N, L)) \cong \operatorname{Ext}(\operatorname{Tor}(M, N), L)
$$

and an abelian group $H$ such that in the following diagram

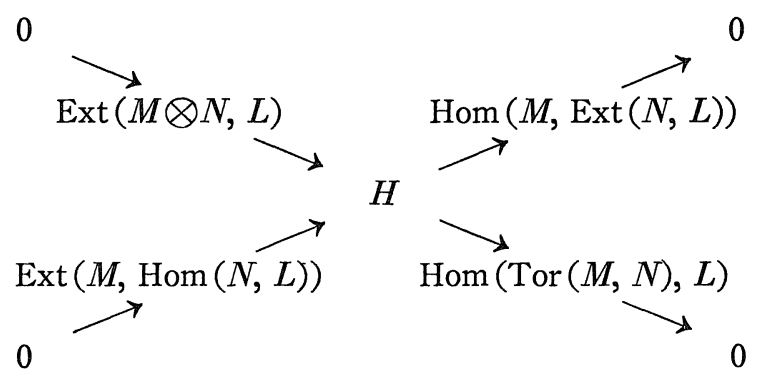

the diagonals are exact.

(1.10) Assume that $M$ is finitely generated. The natural homomorphism $M \otimes \operatorname{Hom}(N, L) \rightarrow \operatorname{Hom}(\operatorname{Hom}(M, N), L)$ admits a factorization (in the diagram below)

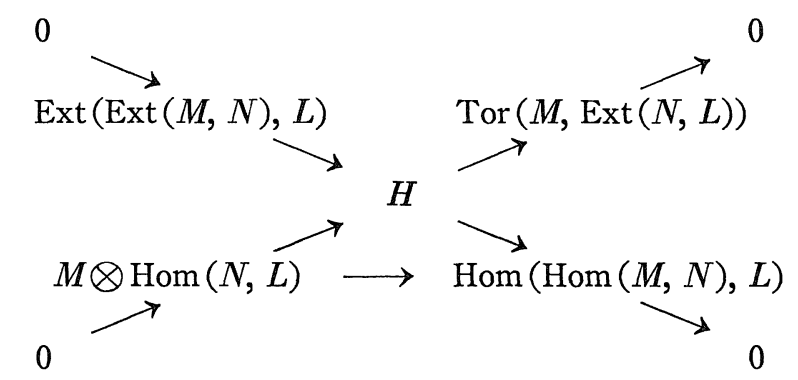

such that the diagonals in the above diagram are exact. Moreover there are isomorphisms

$$
M \otimes \operatorname{Ext}(N, L) \cong \operatorname{Ext}(\operatorname{Hom}(M, N), L)
$$

and

$$
\operatorname{Tor}(M, \operatorname{Hom}(N, L)) \cong \operatorname{Hom}(\operatorname{Ext}(M, N), L)
$$


1.2. Since the global dimension of $Z$ is 1 , the following theorem is obtained as the special case of Roos [14], Theorems 2 and 3. Nevertheless we give a simple proof without using the technique of Roos in our special case $\Lambda=Z$.

Theorem 1 (Roos). Let $\mathscr{A}=\left\{A_{\alpha}\right\}$ be an inverse system of finitely generated abelian groups indexed by a directed set.

i) $\varliminf_{\alpha}{ }^{1} A_{\alpha}$ is injective (as a Z-module), and

ii) $\varliminf_{\alpha}{ }^{p} A_{\alpha}=0 \quad$ for all $p \geqq 2$.

Proof. i) Consider the exact sequence

$$
0 \rightarrow \operatorname{Tor}\left(A_{\alpha}, Z_{q}\right) \rightarrow A_{\alpha} \stackrel{q}{\rightarrow} A_{\alpha} \rightarrow A_{\alpha} \otimes Z_{q} \rightarrow 0
$$

for each integer $q>1$. Since Corollary I.5 implies $\underset{\frac{\lim }{\alpha}}{1} \operatorname{Tor}\left(A_{\alpha}, Z_{q}\right)=$ $\varliminf_{\alpha}^{1}\left(A_{\alpha} \otimes Z_{q}\right)=0$,

$$
q: \varliminf_{\alpha}^{1} A_{\alpha} \rightarrow \varliminf_{\alpha}^{\varliminf_{\alpha}} A_{\alpha}
$$

is an epimorphism. Thus $\lim _{\alpha}^{1} A_{\alpha}$ is divisible by arbitrary non-zero integers and hence injective.

ii) Putting $M=A_{\alpha}$ and $N=L=Z$ in (1.10) we have a (split) exact sequence

$$
0 \rightarrow \operatorname{Ext}\left(\operatorname{Ext}\left(A_{\alpha}, Z\right), Z\right) \rightarrow A_{\alpha} \rightarrow \operatorname{Hom}\left(\operatorname{Hom}\left(A_{\alpha}, Z\right), Z\right) \rightarrow 0
$$

and equalities

$$
\operatorname{Hom}\left(\operatorname{Ext}\left(A_{\alpha}, Z\right), Z\right)=\operatorname{Ext}\left(\operatorname{Hom}\left(A_{\alpha}, Z\right), Z\right)=0 .
$$

Replacing $B_{\alpha}$ and $M$ by $\operatorname{Hom}\left(A_{\alpha}, Z\right)$ and $Z$ in (1.4) respectively we get an exact sequence

$$
\begin{aligned}
0 & \rightarrow \varliminf^{1} \operatorname{Hom}\left(\operatorname{Hom}\left(A_{\alpha}, Z\right), Z\right) \rightarrow \operatorname{Ext}\left(\varliminf_{\longrightarrow} \operatorname{Hom}\left(A_{\alpha}, Z\right), Z\right) \\
& \rightarrow \varliminf_{\lim } \operatorname{Ext}\left(\operatorname{Hom}\left(A_{\alpha}, Z\right), Z\right) \rightarrow \varliminf^{2} \operatorname{Hom}\left(\operatorname{Hom}\left(A_{\alpha}, Z\right), Z\right) \rightarrow 0
\end{aligned}
$$


and isomorphisms

$$
\varliminf^{p} \operatorname{Ext}\left(\operatorname{Hom}\left(A_{\alpha}, Z\right), Z\right) \cong \varliminf^{p+2} \operatorname{Hom}\left(\operatorname{Hom}\left(A_{\alpha}, Z\right), Z\right) \quad \text { for } p \geqq 1 \text {, }
$$

and again replacing them by $\operatorname{Ext}\left(A_{\alpha}, Z\right)$ and $Z$ we get

$$
\varliminf^{p} \operatorname{Ext}\left(\operatorname{Ext}\left(A_{\alpha}, Z\right), Z\right) \cong \varliminf^{p+2} \operatorname{Hom}\left(\operatorname{Ext}\left(A_{\alpha}, Z\right), Z\right) \quad \text { for } p \geqq 1 \text {. }
$$

Making use of the above exact sequence and isomorphisms we have

$$
\varliminf^{p} \operatorname{Hom}\left(\operatorname{Hom}\left(A_{\alpha}, Z\right), Z\right)=\varliminf^{p} \operatorname{Ext}\left(\operatorname{Ext}\left(A_{\alpha}, Z\right), Z\right)=0
$$

for $p \geqq 2$. From this follow the required results

$$
\varliminf_{\alpha}{ }^{p} A_{\alpha}=0 \quad \text { for all } p \geqq 2 \text {. }
$$

\section{Classifying Spaces of Direct Systems of $C W$-complexes}

2.1. Let $I$ be a directed set and $\mathscr{C}=\left\{X_{\alpha}, f_{\beta}^{\alpha}\right\}$ a direct system of based $C W$-complexes and cellular maps over $I$. In [2] we constructed the classifying space $B \mathscr{C}$ of $\mathscr{C}$ which is a based $C W$-complex with the canonical isomorphism

$$
\varliminf_{\alpha} \pi_{n}\left(X_{\alpha}\right) \cong \pi_{n}(B \mathscr{C})
$$

for each degree $n$ (see [2], Corollary 3 and Theorem 1).

Let $X$ and $Y$ be based $C W$-complexes. The smash product $X \wedge Y$ is equipped with the structure of a based $C W$-complex, retopologized by the weak topology in place of the usual topology [15]. In this sense we understand the smash product as a based $C W$-complex. For any based $C W$-complex $Y$ we define a new direct system $\mathscr{C} \wedge Y$ over $I$ (the smash product of $\mathscr{C}$ and $Y$ ) by

$$
\mathscr{C} \wedge Y=\left\{X_{\alpha} \wedge Y, f_{\beta}^{\alpha} \wedge i d\right\}
$$

Making use of [15], Theorem 4.4, we see directly that the classifying space of $\mathscr{C} \wedge Y$ is the smash product of the classifying space $B \mathscr{C}$ and $Y$, i.e., 


$$
B(\mathscr{C} \wedge Y)=B \mathscr{C} \wedge Y
$$

Let $\mathscr{C}=\left\{X_{\alpha}, f_{\beta}^{\alpha}\right\}$ and $\mathscr{C}^{\prime}=\left\{Y_{\alpha}, g_{\beta}^{\alpha}\right\}$ be direct systems over $I$ and $h: \mathscr{C} \rightarrow \mathscr{C}^{\prime}$ a morphism of direct systems. $h$ is a family of cellular maps $h_{\alpha}, \alpha \in I$, satisfying $g_{\beta}^{\alpha} h_{\alpha}=h_{\beta} f_{\beta}^{\alpha}$ for $\alpha \leqq \beta$. For each $n$-simplex $\sigma=\left\{\alpha_{0}\right.$, $\left.\cdots, \alpha_{n}\right\}$ we associate $X_{\sigma}$ and $Y_{\sigma}$ by $X_{\sigma}=X_{\alpha_{0}}$ and $Y_{\sigma}=Y_{\alpha_{0}}$, and $h_{\sigma}: X_{\sigma} \rightarrow$ $Y_{\sigma}$ by $h_{\sigma}=h_{\alpha_{0}}$. As is easily seen, $h_{\sigma} \wedge i d: X_{\sigma} \wedge \triangle^{n,+} \rightarrow Y_{\sigma} \wedge \triangle^{n,+}$ induce a cellular map

$$
B h: B \mathscr{C} \rightarrow B \mathscr{C}^{\prime}
$$

Let $\mathscr{C}_{h}^{\prime} \bigcup_{h} \mathscr{C}$ be the mapping cone of $h: \mathscr{C} \rightarrow \mathscr{C}^{\prime}$ defined by

$$
\mathscr{C}^{\prime} \bigcup_{h} C \mathscr{C}=\left\{Y_{\alpha} \bigcup_{h_{\alpha}} C X_{\alpha}, g_{\beta}^{\alpha} \bigcup C f_{\beta}^{\alpha}\right\}
$$

It is easy to verify that the classifying space of $\mathscr{C}^{\prime} \bigcup_{h} C \mathscr{C}$ is the mapping cone of $B h$, i.e.,

$$
B\left(\mathscr{C}^{\prime} \bigcup_{h} C \mathscr{C}\right)=B \mathscr{C}^{\prime} \bigcup_{B h} C(B \mathscr{C})
$$

Let $X$ be a (connected) based $C W$-complex. We say a direct system $\mathscr{C}=\left\{X_{\alpha}, f_{\beta}^{\alpha}\right\}$ over $I$ is derived from $X$ if

$$
X_{\alpha}=X \text { for } \alpha \in I \text { and } f_{\beta}^{\alpha}=i d \text { for } \alpha \leqq \beta
$$

and denote it by $\mathscr{C}_{X}$. The canonical projection $\sigma: B \mathscr{C}_{X} \rightarrow X$ induced by the projections $X_{\sigma} \wedge \triangle^{n,+} \rightarrow X_{\sigma}$ for $n$-simplexes $\sigma$ is a homotopy equivalence (cf., [2], Proposition 5). Let $\iota_{\alpha}: X \rightarrow B \mathscr{C}_{X}$ be the injection $X=X_{\alpha}$ $C\left(B \mathscr{C}_{X}\right)_{0} \subset B \mathscr{C}_{X}$. Since $\sigma \cdot \iota_{\alpha}=i d$ for each $\alpha \in I$,

(2.6) $\iota_{\alpha}$ is a homotopy equivalence with the homotopy inverse $\varpi$.

2.2. Order the set of all positive integers by divisibility and denote its ordered set by $J$. Obviously $J$ is directed. Since all positive integers are divisible by 1,1 is the initial object of $J$.

Let $M_{q}=S^{1} \bigcup_{q} C S^{1}$ be the co-Moore space of type $\left(Z_{q}, 2\right)$ where $q$ is a positive integer and $q: S^{1} \rightarrow S^{1}$ denotes the map of degree $q$ given by 
$q\{t\}=\{q t\}$ for $\{t \bmod 1\} \in S^{1}$. Denote by

$$
i_{q}: S^{1} \rightarrow M_{q} \text { and } \pi_{q}: M_{q} \rightarrow S^{2}
$$

the canonical inclusion and the map collapsing $S^{1}$ to a point. Let $a, b, q$ and $r$ be integers such that $a r=b q$ and $q, r \geqq 1$. We define a cellular map $\bar{a}: M_{q} \rightarrow M_{r}$ as follows [1]:

$$
\bar{a}=b \cup C a: M_{q}=S^{1} \bigcup_{q} C S^{1} \rightarrow M_{r}=S^{1} \bigcup_{r} C S^{1} .
$$

Consider the following commutative diagram

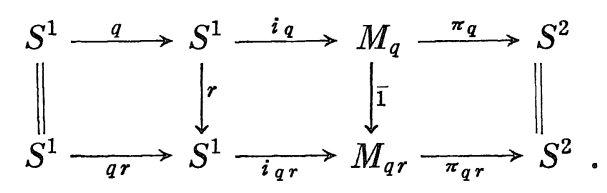

$\left\{S^{1}, f_{q r}^{q}=r\right\}$ and $\left\{M_{q}, \bar{f}_{q r}^{q}=\overline{1}\right\}$ form direct systems of based $C W$-complexes and cellular maps over $J$. We denote by $S_{l}$ and $\check{M}$ the classifying spaces of them respectively, i.e.,

$$
S_{l}=B\left\{S^{1}, f_{q r}^{q}=r\right\} \text { and } \check{M}=B\left\{M_{q}, \bar{f}_{q r}^{q}=\overline{1}\right\} \text {. }
$$

In the next section we will determine the ordinary cohomology groups of $S_{l}$ and $\check{M}$.

The above diagram induces a sequence of direct systems

$$
\mathscr{C}_{S^{1}} \stackrel{j}{\longrightarrow}\left\{S^{1}, f_{q r}^{q}=r\right\} \stackrel{i}{\longrightarrow}\left\{M_{q}, \bar{f}_{q r}^{q}=\overline{1}\right\} \stackrel{\pi}{\longrightarrow} \mathscr{C}_{S^{2}}
$$

where $\mathscr{C}_{S^{k}}, k=1$ and 2 , are direct systems over $J$ derived from $S^{k}$, (2.5). By (2.4) we remark

$$
\check{M}=S_{l} \bigcup_{B j} C\left(B \mathscr{C}_{S^{1}}\right)
$$

Using the maps $c_{1}$ and $\sigma$ of the homotopy equivalences (2.6) we define cellular maps

$$
l: S^{1} \rightarrow S_{l}, \tilde{\imath}: S_{l} \rightarrow \check{M} \text { and } \tilde{\pi}: \check{M} \rightarrow S^{2}
$$

as follows: 


$$
\begin{gathered}
l=B j \cdot \iota_{1}: S^{1} \rightarrow B \mathscr{C}_{S^{1}} \rightarrow S_{l}, \\
\tilde{\imath}=B i: S_{l} \rightarrow \check{M}
\end{gathered}
$$

and

$$
\tilde{\pi}=\sigma \cdot B \pi: \check{M} \rightarrow B \mathscr{C}_{S^{2}} \rightarrow S^{2}
$$

A four-term sequence $X \stackrel{f}{\longrightarrow} Y \stackrel{g}{\longrightarrow} Z \stackrel{h}{\longrightarrow} S X$ of based $C W$-complexes is said to be a cofibration sequence if we have a homotopy commutative diagram

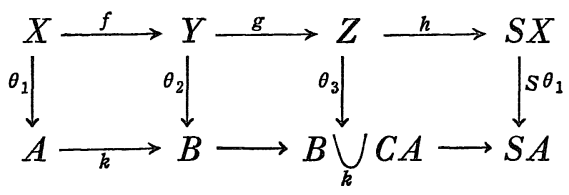

in which the vertical maps are homotopy equivalences.

By means of (2.2), (2.9) and (2.10) we have

Lemma 1. $S^{1} \stackrel{l}{\longrightarrow} S_{l} \stackrel{\tilde{\imath}}{\longrightarrow} \check{M} \stackrel{\tilde{\pi}}{\longrightarrow} S^{2}$ is a cofibration sequence.

\section{Topological Abelian Groups $h(X)$}

3.1. Let $h$ be a (general reduced) cohomology theory, $X$ a based $C W$-complex and $\mathfrak{U}_{X}=\left\{X^{\lambda}\right\}$ be the set of all finite subcomplexes of $X$ ordered by inclusions. $\mathfrak{u}_{X}$ is directed.

For each degree $n$ we define subgroups $F^{\lambda} h^{n}(X)$ of $h^{n}(X)$ by

$$
F^{\lambda} h^{n}(X)=\operatorname{Ker}\left\{h^{n}(X) \rightarrow h^{n}\left(X^{\lambda}\right)\right\}
$$

This inverse system $\left\{F^{\lambda} h^{n}(X)\right\}$ topologizes the abelian group $h^{n}(X)$, the subgroups $F^{\lambda} h^{n}(X)$ being taken as neighborhoods of zero. Denote by $S^{n}(X)$ the closure of zero in $h^{n}(X)$, i.e.,

$$
S^{n}(X)=\bigcap_{\lambda} F^{\lambda} h^{n}(X)
$$

In general $S^{n}(X)$ may contain some elements other than zero, whence 
$h^{n}(X)$ is not necessarily Hausdorff. We say $h(X)$ is Hausdorff when $h^{n}(X)$ is so for each degree $n$ and further $h$ is Hausdorff when $h(X)$ is so for any based $C W$-complex $X$.

The completion of $h^{n}(X)$ for this topology is denoted by $h^{n}(X)^{\wedge}$. In other words,

$$
h^{n}(X)^{\wedge}=\varliminf_{\lambda} h^{n}(X) / F^{\lambda} h^{n}(X)
$$

$h^{n}(X)^{\wedge}$ is topologized by the inverse limit topology. The projections $u_{\lambda}$ : $h^{n}(X) \rightarrow h^{n}(X) / F^{\lambda} h^{n}(X)$ induce a natural homomorphism. We can easily verify that

(3.2) $u$ is a continuous and open map.

$$
u: h^{n}(X) \rightarrow h^{n}(X)^{\wedge}
$$

Similarly $\varliminf_{\lambda} h^{n}\left(X^{\lambda}\right)$ is topologized by the inverse limit topology and the natural homomorphism

$$
\pi=\varliminf_{\lambda} i_{\lambda}^{*}: h^{n}(X) \rightarrow \varliminf_{\lambda} h^{n}\left(X^{\lambda}\right)
$$

is continuous where $i_{\lambda}: X^{\lambda} \subset X$ are the inclusions. By [9], Theorem 1.8, we notice that

(3.4) $\pi: h^{n}(X) \rightarrow \underset{\varliminf_{i}}{\lim _{\lambda}} h^{n}\left(X^{\lambda}\right)$ is an epimorphism for each $n$ if $h$ is additive.

Consider the following diagram

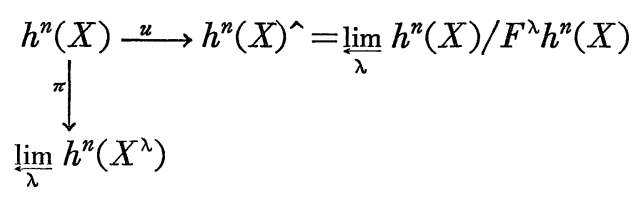

$\pi$ admits a factorization $\hat{\pi} \cdot u$ where the homomorphism $\hat{\pi}: \varliminf_{\lambda} h^{n}(X) /$ $F^{\lambda} h^{n}(X) \rightarrow \varliminf_{\lambda} h^{n}\left(X^{\lambda}\right)$ is induced by the inclusions $i_{\lambda}: X^{\lambda} \subset X$. Obviously $\hat{\pi}$ is a monomorphism. (3.4) implies that $\hat{\pi}$ is an epimorphism and hence it is an isomorphism. Therefore we have 
(3.5) $u: h^{n}(X) \rightarrow h^{n}(X)^{\wedge}$ is an epimorphism for each $n$ if $h$ is additive.

By (3.2), (3.4) and (3.5) we have

Proposition 2. Let $h$ be an additive cohomology theory and $X$ a based CW-complex. Fix a degree $n$. The following conditions are equivalent:

i) $S^{n}(X)=\bigcap_{\lambda} F^{\lambda} h^{n}(X)=\{0\}$,

ii) $h^{n}(X)$ is Hausdorff,

iii) $h^{n}(X)$ is complete and Hausdurff, i.e., $u: h^{n}(X) \rightarrow h^{n}(X)^{\wedge}$ is a homeomorphism,

iv) $\pi: h^{n}(X) \rightarrow \underset{i}{\varliminf_{\lambda}} h^{n}\left(X^{\lambda}\right)$ is an isomorphism.

3.2. Suppose that a cohomology theory $h$ is additive. We observe the spectral sequence $\left\{E_{r}\right\}$ associated with $h^{*}(X)$ such that $E_{2}^{p, q}=$ $\varliminf_{\lambda} h^{p} h^{q}\left(X^{\lambda}\right)$, [2], Theorem 2. The edge homomorphism of the spectral sequence $\left\{E_{r}\right\}$ is the composite

$$
h^{n}(X) \rightarrow E_{\infty}^{0, n} \subset E_{2}^{0, n}=\varliminf_{\lambda} h^{n}\left(X^{\lambda}\right)
$$

and clearly coincides with the natural homomorphism $\pi$. If our spectral sequence collapses, it is strongly convergent by [2], Proposition 9. By standard arguments about spectral sequences and Proposition 2 we can show

Proposition 3. Let $h$ be an additive cohomology theory and $X$ a based $C W$-complex. If $\varliminf_{\lambda} h^{p}\left(X^{\lambda}\right)=0$ for all $n$ and $p \geqq 1$, then $h(X)$ is Hausdorff.

Putting the above proposition and Proposition I.6 together we have

Corollary 4. Let $h$ be an additive $(F)$-cohomology theory. Then $h$ is Hausdorff.

If $h$ is additive and of finite type, then we have a short exact 
sequence

$$
0 \rightarrow \varliminf_{\lambda}^{1} h^{n-1}\left(X^{\lambda}\right) \rightarrow h^{n}(X) \stackrel{\pi}{\rightarrow} \varliminf_{\lambda} h^{n}\left(X^{\lambda}\right) \rightarrow 0
$$

for each degree $n$ (by virtue of Theorem 1, ii)). (Cf., [2], Corollary 12). Hence we get an isomorphism

$$
S^{n}(X) \cong \lim _{\lambda}^{1} h^{n-1}\left(X^{\lambda}\right)
$$

Now we give a characterization of $S^{n}(X)$.

Theorem 2. Let $h$ be an additive cohomology theory of finite type and $X$ a based $C W$-complex. Fix a degree $n$ and take an element $x$ of $h^{n}(X)$.

i) If $x$ is an element of $S^{n}(X)$, then $x$ is divisible in $S^{n}(X)$ by arbitrary non-zero integers. And conversely,

ii) if $x$ is divisible in $h^{n}(X)$ by arbitrary non-zero integers, then $x$ is an element of $S^{n}(X)$.

(Cf., [3]).

Proof. i) Since $S^{n}(X) \cong \varliminf^{1} h^{n-1}\left(X^{\lambda}\right)$ by $(3.7), S^{n}(X)$ is injective by virtue of Theorem $1, \mathrm{i})$. Thus in the group $S^{n}(X)$ the equation $x=$ $m y$ is solvable for any $x \in S^{n}(X)$ and $m \neq 0$.

ii) Suppose that $x \in h^{n}(X)$ is divisible by arbitrary integers $\neq 0$. We denote by $x_{\lambda}$ the restrictions of $x$ to the finite subcomplexes $X^{\lambda}$ of $X$. For each $\lambda x_{\lambda}$ is also divisible in the group $h^{n}\left(X^{\lambda}\right)$. However $h^{n}\left(X^{\lambda}\right)$ is finitely generated. Hence we see immediately

$$
x_{\lambda}=0 \in h^{n}\left(X^{\lambda}\right) \quad \text { for any } \lambda \text {. }
$$

This results that $x$ is an element of $S^{n}(X)$.

Q.E.D.

Theorem 2 means that the subgroup $S^{n}(X)$ has an infinite number of generators if $h^{n}(X)$ is not Hausdorff. Hence we have

Corollary 5. Let $h$ be an additive cohomology theory of finite type and $X$ be a based $C W$-complex such that $h^{n}(X)$ is finitely generated for 
some degree $n$. Then $h^{n}(X)$ is Hausdorff for the same degree $n$.

3.3. Let $h$ be an additive cohomology theory of finite type and $\mathscr{C}=$ $\left\{X_{\alpha}, f_{\beta}^{\alpha}\right\}$ be a direct system of based finite $C W$-complexes and cellular maps. There exists an exact sequence

$$
0 \rightarrow \varliminf_{\alpha}^{1} h^{n-1}\left(X_{\alpha}\right) \rightarrow h^{n}(B \mathscr{C}) \rightarrow \varliminf_{\alpha} h^{n}\left(X_{\alpha}\right) \rightarrow 0
$$

for each degree $n$ (cf., (3.6)) which is implicitly contained in [2], Corollary 12 .

(3.8) allows us to compute the ordinary (reduced) cohomology groups $\tilde{H}^{*}\left(S_{l}\right)$ and $\tilde{H}^{*}(\check{M})$ of the spaces $S_{l}$ and $\check{M}$ of (2.8).

Proposition 6. $\check{M}$ and $S_{l}$ are co-Moore spaces of type $(\hat{Z}, 2)$ and $(\hat{Z} / Z, 2)$ respectively where $\hat{Z}$ is the completion of $Z$ with respect to all of its subgroups.

Proof. Consider the following commutative diagram

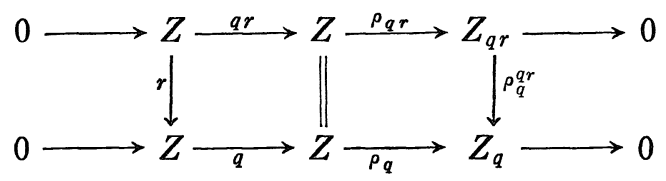

in which $\rho_{q}, \rho_{q r}$ and $\rho_{q}^{q r}$ are the canonical projections. This yields an exact sequence

$$
0 \rightarrow \varliminf_{\frac{l_{j}}{J}}\left(Z, g_{q}^{q r}=r\right) \rightarrow Z \stackrel{\iota}{\rightarrow} \hat{Z} \rightarrow{\frac{\lim _{J}}{J}}^{1}\left(Z, g_{q}^{q r}=r\right) \rightarrow 0
$$

and an equality

$$
\varliminf_{J}^{1}\left(Z_{q}, \rho_{q}^{q r}\right)=0
$$

where the index set $J$ is given in the preceding section. Clearly $\iota$ is the canonical inclusion. Therefore we get

$$
\varliminf_{J}\left(Z, g_{q}^{q r}=r\right)=0 \text { and } \varliminf_{\frac{l^{\prime}}{J}}^{1}\left(Z, g_{q}^{q r}=r\right)=\hat{Z} / Z
$$


Applying (3.8) to $\check{M}$ and $S_{l}$ we have exact sequences

$$
0 \rightarrow \varliminf_{J}^{1} \tilde{H}^{n-1}\left(M_{q}\right) \rightarrow \tilde{H}^{n}(\check{M}) \rightarrow \varliminf_{J} \tilde{H}^{n}\left(M_{q}\right) \rightarrow 0
$$

and

$$
0 \rightarrow \varliminf_{J}^{1}\left(\tilde{H}^{n-1}\left(S^{1}\right), g_{q}^{q r}=r\right) \rightarrow \tilde{H}^{n}\left(S_{l}\right) \rightarrow \varliminf_{J}\left(\tilde{H}^{n}\left(S^{1}\right), g_{q}^{q r}=r\right) \rightarrow 0
$$

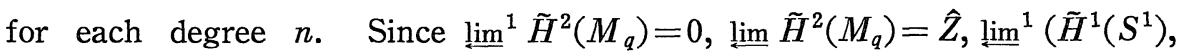
$\left.g_{q}^{q r}=r\right)=\hat{Z} / Z$ and $\varliminf\left(\tilde{H}^{1}\left(S^{1}\right), g_{q}^{q r}=r\right)=0, \check{M}$ and $S_{l}$ are the desirable coMoore spaces.

Q.E.D.

Remark. There is an isomorphism

$$
\varliminf_{\alpha} \tilde{H}_{n}\left(X_{\alpha}\right) \cong \tilde{H}_{n}(B \mathscr{C})
$$

for any direct system $\mathscr{C}=\left\{X_{\alpha}, f_{\beta}^{\alpha}\right\}$ of based $C W$-complexes and cellular maps ([2], Theorem 3). By means of this fact we can show that $S_{l}$ and $\check{M}$ are Moore spaces of type $(Q, 1)$ and $(Q / Z, 1)$ respectively where $Q$ is the field of rational numbers. And we see

$$
\operatorname{Ext}(Q, Z) \cong \hat{Z} / Z \text { and } \operatorname{Ext}(Q / Z, Z) \cong \hat{Z}
$$

because of (1.4).

\section{Cohomology Theories with Coefficients $\hat{Z}$ and $\hat{Z} / Z$}

4.1. Let $h$ be a (general reduced) cohomology theory and $X$ a based $C W$-complex. Since we have the co-Moore spaces $M_{q}, \check{M}$ and $S_{l}$ of type $\left(Z_{q}, 2\right),(\hat{Z}, 2)$ and $(\hat{Z} / Z, 2)$ respectively we can define cohomology theories with coefficients $Z_{q}, \hat{Z}$ and $\hat{Z} / Z$ as follows ( $[11]$ and also [1]):

$$
\begin{gathered}
h^{n}\left(X ; Z_{q}\right)=h^{n+2}\left(X \wedge M_{q}\right), q>1, \\
h^{n}(X ; \hat{Z})=h^{n+2}(X \wedge \check{M}) \text { and } h^{n}(X ; \hat{Z} / Z)=h^{n+2}\left(X \wedge S_{l}\right)
\end{gathered}
$$

for each degree $n$.

We remark that 


$$
\bigvee_{\alpha}\left(X_{\alpha} \wedge Y\right)=\left(\bigvee_{\alpha} X_{\alpha}\right) \wedge Y
$$

This implies that

(4.2) $h\left(; Z_{q}\right), h(; \hat{Z})$ and $h(; \hat{Z} / Z)$ are additive if $h$ is so.

Using the maps $l, \tilde{i}$ and $\tilde{\pi}$ given in (2.10) we define maps

$$
\iota: h^{n}(X) \rightarrow h^{n}(X ; \hat{Z}), \kappa: h^{n}(X ; \hat{Z}) \rightarrow h^{n}(X ; \hat{Z} / Z)
$$

$$
\text { and } \quad \delta: h^{n}(X ; \hat{Z} / Z) \rightarrow h^{n+1}(X)
$$

by

$$
\iota=(1 \wedge \tilde{\pi})^{*} \sigma^{2}, \kappa=(1 \wedge \tilde{\imath})^{*} \text { and } \delta=\sigma^{-1}(1 \wedge l)^{*}
$$

where $\sigma$ is the suspension isomorphism.

From the exact sequence of $h$ associated with the cofibration sequence (Lemma 1):

$$
X \wedge S^{1} \stackrel{1 \wedge l}{\longrightarrow} X \wedge S_{l} \stackrel{1 \wedge \tilde{\imath}}{\longrightarrow} X \wedge \check{M} \stackrel{1 \wedge \tilde{\pi}}{\longrightarrow} X \wedge S^{2},
$$

we obtain the following exact sequence

$$
\longrightarrow h^{n}(X) \stackrel{\iota}{\longrightarrow} h^{n}(X ; \hat{Z}) \stackrel{\kappa}{\longrightarrow} h^{n}(X ; \hat{Z} / Z) \stackrel{\delta}{\longrightarrow} h^{n+1}(X) \longrightarrow
$$

corresponding to the coefficients sequence $0 \rightarrow Z \rightarrow \hat{Z} \rightarrow \hat{Z} / Z \rightarrow 0$.

4.2. From now on until the end of this section we shall understand that $h$ is an additive cohomology theory of finite type. Let $X$ be a based $C W$-complex and $\mathfrak{U}_{X}=\left\{X^{\lambda}\right\}$ the set of all finite subcomplexes of $X$ ordered by inclusions.

First we study properties of $h\left(; Z_{q}\right), q>1$.

Proposition 7. Let $h$ be an additive cohomology theory of finite type and $X$ a based $C W$-complex. $h^{n}\left(X ; Z_{q}\right)$ is a profinite abelian group for each degree $n$. More precisely the natural map

$$
\pi: h^{n}\left(X ; Z_{q}\right) \rightarrow \varliminf_{\lambda} h^{n}\left(X^{\lambda} ; Z_{q}\right)
$$


is a homeomorphism.

Proof. By the assumptions on $h, h\left(; Z_{q}\right)$ becomes an additive $(F)$ cohomology theory (see I. 2.3.). Hence we see

$$
\varliminf_{\frac{\lim }{\lambda}}^{p} h^{n}\left(X^{\lambda} ; Z_{q}\right)=0 \quad \text { for } \quad p \geqq 1
$$

by Corollary I.5. The natural map

$$
u: h^{n}\left(X ; Z_{q}\right) \rightarrow h^{n}\left(X ; Z_{q}\right)^{\wedge}=\varliminf_{\lambda} h^{n}\left(X ; Z_{q}\right) / F^{\lambda} h^{n}\left(X Z_{q}\right)
$$

is a homeomorphism because of (3.2) and (3.5). Thus $h^{n}\left(X ; Z_{q}\right)$ is a profinite abelian group as $h^{n}\left(X ; Z_{q}\right) / F^{\lambda} h^{n}\left(X ; Z_{q}\right)$ is finite. Since a profinite abelian group is compact Hausdorff $([3]$ and also $[\mathrm{I}])$, the continuous map $\pi$ is also a homeomorphism.

Q.E.D.

By (2.2) we recall

$$
Y \wedge \check{M}=B\left\{Y \wedge M_{q}, f_{q r}^{q}=i d \wedge \overline{1}\right\}
$$

for any based $C W$-complex $Y$. Applying (3.8) to $Y \wedge \check{M}$ we get an exact sequence

$$
0 \rightarrow \varliminf_{q}^{1 m^{1}} h^{n-1}\left(Y ; Z_{q}\right) \rightarrow h^{n}(Y ; \hat{Z}) \stackrel{\rho}{\rightarrow} \varliminf_{q} h^{n}\left(Y ; Z_{q}\right) \rightarrow 0
$$

for each $n$ when $Y$ is finite. Now, by Corollary I.5 we obtain an isomorphism

$$
\rho: h^{n}(Y ; \hat{Z}) \rightarrow \varliminf_{q} h^{n}\left(Y ; Z_{q}\right)
$$

for any based finite $C W$-complex $Y$.

For each $n\left\{h^{n}\left(X^{\lambda} ; Z_{q}\right)\right\}$ forms an inverse system of finite abelian groups indexed by pairs $(\lambda, q)$. By $[8]$, Theorem 3 (see also Theorem I.1) there exist two strongly convergent spectral sequences $\left\{E_{r}\right\}$ and $\left\{\bar{E}_{r}\right\}$ associated with $\varliminf_{\lambda, q} * h^{n}\left(X^{\lambda} ; Z_{q}\right)$ such that

$$
E_{2}^{p, s}=\varliminf_{\lambda}^{\varliminf_{i}^{p}} \varliminf_{q}^{l i m} s h^{n}\left(X^{\lambda} ; Z_{q}\right) \text { and } \bar{E}_{2}^{p, s}=\varliminf_{q}^{\varliminf_{i}^{p}} \varliminf_{\lambda}^{l i m} s h^{n}\left(X^{\lambda} ; Z_{q}\right) .
$$


Since Corollary I.5 implies

$$
E_{2}^{p, s}=\bar{E}_{2}^{p, s}=0 \quad \text { unless } s=0
$$

we see

$$
E_{2}^{p .0}=E_{\infty}^{p, 0} \cong \varliminf_{\lambda, q}^{p} h^{n}\left(X^{\lambda} ; Z_{q}\right) \text { and } \bar{E}_{2}^{p, 0}=\bar{E}_{\infty}^{p, 0} \cong \varliminf_{\lambda, q}^{p} h^{n}\left(X^{\lambda} ; Z_{q}\right) .
$$

And using Corollary I.5 again we get

$$
E_{2}^{p, 0}=\bar{E}_{2}^{p, 0}=0 \quad \text { for all } p \geqq 1 .
$$

On the other hand, it follows from (4.5) and Proposition 7 that

$$
E_{2}^{p, 0} \cong \varliminf_{\varliminf_{i}}^{p} h^{n}\left(X^{\lambda} ; \hat{Z}\right) \text { and } \bar{E}_{2}^{p, 0} \cong \varliminf_{q}^{\varliminf_{m}^{p}} h^{n}\left(X ; Z_{q}\right) \text {. }
$$

In particular we obtain

$$
\varliminf_{\lambda} h^{n}\left(X^{\lambda} ; \hat{Z}\right)=\varliminf_{q}^{p} h^{n}\left(X ; Z_{q}\right)=0 \quad \text { for } p \geqq 1 \text {. }
$$

Proposition 8. Let $h$ be an additive cohomology theory of finite type and $X$ a based $C W$-complex. $h(; \hat{Z})$ is Hausdorff and moreover

$$
\rho: h^{n}(X ; \hat{Z}) \rightarrow \underset{q}{\varliminf_{q}} h^{n}\left(X ; Z_{q}\right)
$$

is an isomorphism for each $n$.

Proof. The first part follows immediately from (4.6) and Proposition 3. Applying the spectral sequence of [2], Proposition 10 to $\left\{X \wedge M_{q}, f_{q r}^{q}\right.$ $=i d \wedge \overline{1}\}$, the second part is also obtained by means of (4.6) and [2], Proposition 9.

4.3. Now we study universal coefficient formulas for $h(; \hat{Z})$ and $h(; \hat{Z} / Z)$. Let $h$ be an additive cohomology theory of finite type and $X$ a based $C W$-complex. Assume that $h^{n}(X)$ is a finitely generated abelian group for each $n . h(X)$ is Hausdorff by Corollary 5 .

Consider the exact universal coefficient sequence

$$
0 \rightarrow h^{n}(X) \otimes Z_{q} \rightarrow h^{n}\left(X ; Z_{q}\right) \rightarrow \text { Tor }\left(h^{n+1}(X), Z_{q}\right) \rightarrow 0
$$


Since $h^{n}(X) \otimes Z_{q}$ and $\operatorname{Tor}\left(h^{n}(X), Z_{q}\right)$ are finite we have

$$
\varliminf_{q}^{p}\left(h^{n}(X) \otimes Z_{q}\right)=\varliminf_{q}^{\varliminf^{p}} \text { Tor }\left(h^{n}(X), Z_{q}\right)=0 \quad \text { for } p \geqq 1 .
$$

By means of (1.6) we see that the tensor and torsion products, $\otimes$ and Tor, commute with the inverse limits in the following cases:

$$
\varliminf_{q}\left(h^{n}(X) \otimes Z_{q}\right) \cong h^{n}(X) \otimes \hat{Z} \text { and } \varliminf_{q} \operatorname{Tor}\left(h^{n+1}(X), Z_{q}\right) \cong \operatorname{Tor}\left(h^{n+1}(X), \hat{Z}\right) .
$$

On the other hand Proposition 8 shows

$$
\varliminf_{q} h^{n}\left(X ; Z_{q}\right) \cong h^{n}(X ; \hat{Z}) .
$$

Then the above universal coefficient sequence yields an exact sequence

$$
0 \rightarrow h^{n}(X) \otimes \hat{Z} \stackrel{\hat{\iota}}{\rightarrow} h^{n}(X ; \hat{Z}) \rightarrow \operatorname{Tor}\left(h^{n+1}(X), \hat{Z}\right) \rightarrow 0 .
$$

However $\hat{Z}$ is torsion free. Hence we get the first formula

$$
h^{n}(X ; \hat{Z}) \cong h^{n}(X) \otimes \hat{Z} \quad \text { for each } n
$$

Next, in the following commutative diagram

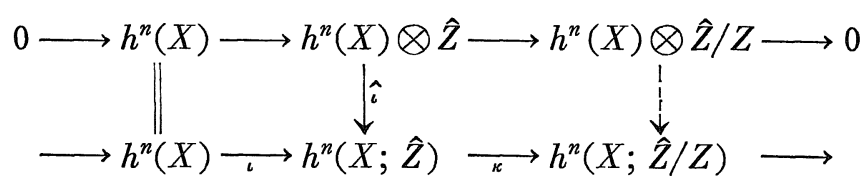

the upper row is exact by the fact that $\hat{Z} / Z$ is torsion free and the lower one is so by (4.4). Then the right (dotted) vertical map is induced by the central one $\hat{\iota}$. Using the above formula (4.7) we see that $\iota: h^{n}(X) \rightarrow$ $h^{n}(X ; \hat{Z})$ is a monomorphism for each $n$. Thus

$$
0 \rightarrow h^{n}(X) \stackrel{\iota}{\rightarrow} h^{n}(X ; \hat{Z}) \stackrel{\kappa}{\rightarrow} h^{n}(X ; \hat{Z} / Z) \rightarrow 0
$$

is an exact sequence for each $n$. Applying "five lemma" in the above commutative diagram we get the second formula

$$
h^{n}(X ; \hat{Z} / Z) \cong h^{n}(X) \otimes \hat{Z} / Z \quad \text { for each } n
$$

Here we summarize results of (4.7) and (4.9). 
Proposition 9. Let $h$ be an additive cohomology theory of finite type and $X$ be a based CW-complex such that $h^{n}(X)$ is a finitely generated abelian group for each $n$. There are isomorphisms

$$
h^{n}(X ; \hat{Z}) \cong h^{n}(X) \otimes \hat{Z} \text { and } h^{n}(X ; \hat{Z} / Z) \cong h^{n}(X) \otimes \hat{Z} / Z
$$

for all $n$.

4.4. Let $X$ be a based $C W$-complex and $X^{\lambda}$ run over all finite subcomplexes of $X$. (4.8) yields an exact sequence

$$
\begin{aligned}
0 \rightarrow & \varliminf_{\bar{\lambda}} h^{n}\left(X^{\lambda}\right) \rightarrow \varliminf_{\lambda} h^{n}\left(X^{\lambda} ; \hat{Z}\right) \\
& \rightarrow \underset{\frac{l_{\lambda}}{\lambda}}{n} h^{n}\left(X^{\lambda} ; \hat{Z} / Z\right) \rightarrow \varliminf_{\bar{\lambda}}^{1} h^{n}\left(X^{\lambda}\right) \rightarrow 0
\end{aligned}
$$

and equalities

$$
\varliminf_{\lambda} h^{n}\left(X^{\lambda} ; \hat{Z}\right)=\varliminf_{\frac{l_{i}}{p}} h^{n}\left(X^{\lambda} ; \hat{Z} / Z\right)=0 \quad \text { for all } p \geqq 1 \text {, }
$$

by virtue of Theorem 1, ii) and (4.6). By (4.11) and Proposition 3 we have

Proposition 10. Let $h$ be an additive cohomology theory of finite type. Then $h(; \hat{Z} / Z)$ is Hausdorff.

(4.10) combined with Propositions 8 and 10 shows that there is an exact sequence (cf., [10])

$$
0 \rightarrow \varliminf_{\lambda} h^{n}\left(X^{\lambda}\right) \rightarrow h^{n}(X ; \hat{Z}) \rightarrow h^{n}(X ; \hat{Z} / Z) \rightarrow \varliminf_{\lambda}^{\varliminf_{i}^{1}} h^{n}\left(X^{\lambda}\right) \rightarrow 0 .
$$

Theorem 3. Let $h$ be an additive cohomology theory of finite type and $X$ a based $C W$-complex. The following sequence

$$
h^{n-1}(X ; \hat{Z} / Z) \stackrel{\delta}{\rightarrow} h^{n}(X) \stackrel{\pi}{\rightarrow} \varliminf_{\lambda} h^{n}\left(X^{\lambda}\right) \rightarrow 0
$$

is exact for each degree $n$ where $X^{\lambda}$ runs over all finite subcomplexes of $X$.

Proof. Consider the following commutative diagram 


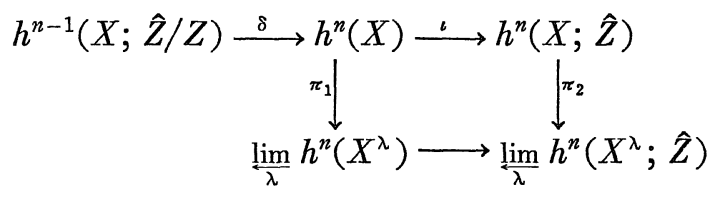

in which the upper row is exact by (4.4). The left vertical map $\pi_{1}$ is an epimorphism by (3.4) and the right one $\pi_{2}$ is an isomorphism by Proposition 8. Moreover $\iota_{\lambda}: h^{n}\left(X^{\lambda}\right) \rightarrow h^{n}\left(X^{\lambda} ; \hat{Z}\right)$ is a monomorphism because of (4.8). Therefore the bottom horizontal map

$$
\varliminf_{\bar{\lambda}} c_{\lambda}: \lim _{\bar{\lambda}} h^{n}\left(X^{\lambda}\right) \rightarrow \varliminf_{\frac{1}{\lambda}} h^{n}\left(X^{\lambda} ; \hat{Z}\right)
$$

is also so. By chasing the above diagram we get the required exact sequence.

Q.E.D.

As an immediate corollary of the above theorem we have

Corollary 11. Let $h$ and $X$ be as in the above theorem. Then

$$
S^{n}(X)=\operatorname{Im}\left\{\delta: h^{n-1}(X ; \hat{Z} / Z) \rightarrow h^{n}(X)\right\} \quad \text { for each } n
$$

4.5. Here we summarize some criteria for the absence of elements of $S^{n}(X)$ for $h$ being an additive cohomology theory of finite type.

Theorem 4. Let $h$ be an additive cohomology theory of finite type and $X$ a based $C W$-complex. Fix a degree $n$ and let $X^{\lambda}$ run over all finite subcomplexes of $X$. The following conditions are equivalent:

i) $h^{n}(X)$ is Hausdorff,

ii) $\varliminf_{\lambda}^{1} h^{n-1}\left(X^{\lambda}\right)=0$,

iii) $\delta: h^{n-1}(X ; \hat{Z} / Z) \rightarrow h^{n}(X)$ is trivial.

Theorem 5. Let $h$ be as in the above theorem. Let $X$ be a based $C W$-complex with finite skeletons and $\left\{E_{r}\right\}$ be the Atiyah-Hirzebruch spectral sequence of $h$ associated with the skeleton filtration $\left\{X^{p}\right\}_{p \geqq 0}$. The following conditions iv)-vi) are also equivalent to the conditions i)-iii) of the above theorem: 
iv) the inverse system $\left\{h^{n-1}\left(X^{p}\right)\right\}_{p \geqq 0}$ satisfies the Mittag-Leffler condition $(M L)$,

v) for each $p$ there exists $r_{0}=r_{0}(p, n)<\infty$ such that

$$
E_{r_{0}}^{p, n-p-1}=E_{r}^{p, n-p-1} \quad \text { for all } r, r_{0} \leqq r<\infty,
$$

vi) for each $p$ there exists $r_{0}=r_{0}(p, n)<\infty$ such that

$$
E_{\infty}^{p, n-p-1}=E_{r_{0}}^{p, n-p-1} \text {. }
$$

(Cf., $[3]$ and $[13])$.

Proof. The equivalence of iv)-vi) is implicit in [2], §3 (combine Lemmas 6 and 7, i) and ii) with Milnor's short exact sequence). On the other hand, according to the result of Gray [12] it is equivalent to the vanishing of $\varliminf_{p}{ }^{1} h^{n-1}\left(X^{p}\right)$ that the inverse system $\left\{h^{n-1}\left(X^{p}\right)\right\}_{p \geqq 0}$ of finitely generated abelian groups satisfies $(M L)$. Thus ii) is equivalent to iv).

\section{5. (Q)-Cohomology Theories}

5.1. Let $Q$ be the field of rational numbers. We call a cohomology theory $h$ is a $(Q)$-cohomology theory when $h^{n}\left(S^{0}\right)$ is a $Q$-module for each degree $n$. This condition is equivalent to say that $h^{n}\left(S^{0} ; Z_{q}\right)=0$ for all $q>1$ and $n$. Let $h$ be an additive $(Q)$-cohomology theory, $X$ a based $C W$-complex and $\mathfrak{u}_{X}=\left\{X^{\lambda}\right\}$ be the set of all finite subcomplexes of $X$. Obviously $h^{n}\left(X^{\lambda} ; Z_{q}\right)=0$ for the finite subcomplexes $X^{\lambda}$. By Proposition 3 it is straightforward to see that

$$
h^{n}\left(X ; Z_{q}\right)=0 \quad \text { for all } q>1 \text { and } n .
$$

Thus

(5.1) $h^{n}(X)$ is a Q-module for any based $C W$-complex $X$ if $h$ is an additive (Q)-cohomology theory.

Let $h$ be a $(Q)$-cohomology theory. We define a cohomology theory $h_{H}$ by 


$$
h_{H}^{n}(\quad)=\prod_{k+l=n} \tilde{H}^{k}\left(\quad ; h^{l}\left(S^{0}\right)\right)
$$

where $\tilde{H}^{*}$ is the ordinary (reduced) cohomology theory. Since $h_{H}^{n}\left(S^{0}\right) \cong$ $h^{n}\left(S^{0}\right)$ for each $n, h_{H}$ becomes a $(Q)$-cohomology theory. Dold [11] showed that

(5.2) there exists a natural equivalence

$$
T: h \rightarrow h_{H}
$$

defined on the category of based finite CW-complexes.

Let $X$ be a based $C W$-complex and $X^{\lambda}$ run over all finite subcomplexes of $X$. Using (5.2) and the universal coefficient theorem for $\tilde{H}^{*}$ we have natural isomorphisms

$$
h^{n}\left(X^{\lambda}\right) \cong \prod_{k+l=n} \tilde{H}^{k}\left(X^{\lambda} ; h^{l}\left(S^{0}\right)\right) \cong \prod_{k+l=n} \tilde{H}^{k}\left(X^{\lambda}\right) \otimes h^{l}\left(S^{0}\right)
$$

for all $n$. We remark that $h^{l}\left(S^{0}\right)$ is injective (as a $Z$-module). The natural homomorphisms

$$
\begin{aligned}
\tilde{H}^{k}\left(X^{\lambda}\right) \otimes h^{l}\left(S^{0}\right) \cong & H^{k}\left(X^{\lambda}\right) \otimes \operatorname{Hom}\left(Z, h^{l}\left(S^{0}\right)\right) \\
& \rightarrow \operatorname{Hom}\left(\operatorname{Hom}\left(\tilde{H}^{k}\left(X^{\lambda}\right), Z\right), h^{l}\left(S^{0}\right)\right)
\end{aligned}
$$

are isomorphisms by (1.10). And replacing $B_{\lambda}$ and $M$ by $\operatorname{Hom}\left(\tilde{H}^{k}\left(X^{\lambda}\right), Z\right)$ and $h^{l}\left(S^{0}\right)$ in (1.4) respectively, we see immediately that

$$
\varliminf_{\frac{\lim }{\lambda}}^{p} \operatorname{Hom}\left(\operatorname{Hom}\left(\tilde{H}^{k}\left(X^{\lambda}\right), Z\right), h^{l}\left(S^{0}\right)\right)=0 \quad \text { for all } \quad p \geqq 1
$$

Consequently we obtain

$$
\begin{aligned}
& \varliminf_{\lambda} h^{p}\left(X^{\lambda}\right) \cong \prod_{k+l=n} \varliminf_{\lambda} \bar{m}^{p}\left(\tilde{H}^{k}\left(X^{\lambda}\right) \otimes h^{l}\left(S^{0}\right)\right) \\
& \cong \prod_{k+l=n} \lim _{\grave{\lambda}}^{p} \operatorname{Hom}\left(\operatorname{Hom}\left(\tilde{H}^{k}\left(X^{\lambda}\right), Z\right), h^{l}\left(S^{0}\right)\right)=0
\end{aligned}
$$

for $p \geqq 1$, when $h$ is a $(Q)$-cohomology theory.

Using (5.3) and Proposition 3 we have 
Proposition 12. Let $h$ be an additive (Q)-cohomology theory. Then $h$ is Hausdorff.

Let $h$ be an additive cohomology theory of finite type. By (4.9) $h^{n}\left(S^{0} ; \hat{Z} / Z\right) \cong h^{n}\left(S^{0}\right) \otimes \hat{Z} / Z$, and hence it is a $Q$-module. Thus $h(; \hat{Z} / Z)$ is an additive $(Q)$-cohomology theory. And this means that the above proposition contains Proposition 10 as a corollary.

The following theorem is a generalization of Dold's Theorem in the sense that the finiteness restriction on based $C W$-complexes is removed.

Theorem 6. Let $h$ be an additive (Q)-cohomology theory and $X$ a based $C W$-complex. There exists a natural isomorphism

$$
h^{n}(X) \cong \prod_{k+l=n} \tilde{H}^{k}\left(X ; h^{l}\left(S^{0}\right)\right) \quad \text { for each degree } n .
$$

Proof. By (5.2) there are natural isomorphisms

$$
T_{\lambda}^{n}: h^{n}\left(X^{\lambda}\right) \rightarrow h_{H}^{n}\left(X^{\lambda}\right)
$$

for all finite subcomplexes $X^{\lambda}$ of $X$. These yield a natural isomorphism

$$
\varliminf_{\lambda} h^{n}\left(X^{\lambda}\right) \cong \varliminf_{\lambda} h_{H}^{n}\left(X^{\lambda}\right)
$$

Both $h$ and $h_{H}$ are additive $(Q)$-cohomology theories. Therefore we get a natural isomorphism

$$
\widetilde{T}^{n}: h^{n}(X) \rightarrow h_{H}^{n}(X)=\prod_{k+l=n} \tilde{H}^{k}\left(X ; h^{l}\left(S^{0}\right)\right)
$$

by virtue of Proposition 12 .

Q.E.D.

Remark. Since an additive homology theory commutes with the direct limits (see [2], Theorem 3), the dual case of the above theorem is also valid.

As a corollary of Theorem 6 we have

Corollary 13. Let $h$ be an additive cohomology theory of finite type and $X$ a based $C W$-complex. There exists a natural isomorphism 


$$
h^{n}(X ; \hat{Z} / Z) \cong \prod_{k+l=n} \tilde{H}^{k}\left(X ; h^{l}\left(S^{0}\right) \otimes \hat{Z} / Z\right) \text { for each } n
$$

5.2. Let $A$ be an abelian group and $\mathfrak{U}_{A}=\left\{A^{\lambda}\right\}$ be the family of all finitely generated subgroups (directed by inclusions) of $A$. Obviously $A \cong$ $\varliminf A^{\lambda}$ and hence $A \otimes Q \cong \varliminf_{(}\left(A^{\lambda} \otimes Q\right)$. Since (1.4) shows $\operatorname{Ext}(A \otimes Q, Z)$ $\cong \varliminf_{\lim } \operatorname{Ext}\left(A^{\lambda} \otimes Q, Z\right)$, it follows that $\operatorname{Ext}(A \otimes Q, Z)=0$ if and only if $\operatorname{Ext}\left(A^{\lambda} \otimes Q, Z\right)=0$ for all $\lambda$. Therefore we can show that

$$
\operatorname{Ext}(A \otimes Q, Z)=0 \text { if and only if } A \otimes Q=0,
$$

because $\operatorname{Ext}(Q, Z) \cong \hat{Z} / Z$.

Making use of the universal coefficient theorem between $\tilde{H}^{*}$ and $\tilde{H}_{*}$ and (1.9) we get

$$
\begin{aligned}
\tilde{H}^{n}(X ; \hat{Z} / Z) & \cong \operatorname{Hom}\left(\tilde{H}_{n}(X), \hat{Z} / Z\right) \cong \operatorname{Hom}\left(H_{n}(X), \operatorname{Ext}(Q, Z)\right) \\
& \cong \operatorname{Ext}\left(H_{n}(X) \otimes Q, Z\right)
\end{aligned}
$$

for any based $C W$-complex $X$. Hence we have

Lemma 14. $\tilde{H}_{n}(X) \otimes Q=0$ if and only if $\tilde{H}^{n}(X ; \hat{Z} / Z)=0$.

By Corollary 13 and Lemma 14 we have

Proposition 15. Let $h$ be an additive cohomology theory of finite type. If $X$ is a based $C W$-complex with $\tilde{H}_{*}(X) \otimes Q=0$, then $h^{n}(X ; \hat{Z} / Z)$ $=0$ for all $n$.

Finally, combining Proposition 15 with Theorem 4 we obtain a sufficient condition that $h(X)$ is Hausdorff.

Theorem 7. Let $h$ be an additive cohomology theory of finite type and $X$ a based $C W$-complex. In order that $h(X)$ is Hausdorff it is sufficient that $\tilde{H}_{*}(X) \otimes Q=0$. 


\section{References}

[1]-[8] are listed at the end of paper [I].

[9] Adams, J. F., A variant of E. H. Brown's representability theorem, Topology 10 (1971), 185-198.

[10] Anderson, D. W., Universal coefficient theorems for $k$-theory, mimeographed notes, Berkeley.

[11] Dold, A., Relations between ordinary and extraordinary homology, Colloq. on algebraic topology, Aarhus Univ. (1962), 2-9.

[12] Gray, B. I., Spaces of the same $n$-type, for all $n$, Topology, 5 (1966), 241-243.

[13] Landweber, P. S., On the complex bordism and cobordism of infinite complexes, Bull. of Amer. Math. Soc. 76, (3) (1970) 650-654.

[14] Roos, J.-E., Bidualité et structure des foncteurs dérivés de lim dans la catégorie des modules sur un anneau régulier, C.R. Acad. Sci. Paris, 254 (1962), 1556-1558 and $1720-1722$.

[15] Steenrod, N. E., A convenient category of topological spaces, Michigan Math. J. 14 (1967), 133-152.

[I ] Yosimura, Z., On cohomology theories of infinite CW-complexes, I, Publ. RIMS, Kyoto Univ. 8 (1972/73), 295-310. 